\title{
Interactive comment on "A comparison between Envisat and ICESat sea ice thickness in the Antarctic" by Jinfei Wang et al.
}

\section{Anonymous Referee \#1}

Received and published: 31 March 2020

\section{Review of}

A comparison between Envisat and ICESat sea ice thickness in the Antarctic

by Wang, Jinfei, et al.

Summary: This paper is about a study inter-comparing two Antarctic sea-ice thickness products derived from satellite altimetry. One is using Envisat radar altimetry; the other one is based on ICESat laser altimetry. The comparison focusses on years 2004 through 2008, being limited to the periods during which ICESat's laser was operational. The two products are first compared to sea-ice thickness values derived from sea-ice draft observation of a few upward looking sonars (ULS) in the Weddell Sea. This comparison suggests a better agreement between ICESat and ULS sea-ice thickness. 
The core of the paper deals with the inter-comparison between the two satellite-based sea-ice thickness products using standard statistical metrics such as mean, difference, RMSD and correlation. The study shows maps, histograms and scatterplots and summarizes results in two tables. The comparison is carried out for Antarctica as a whole but also broken down to the commonly used sub-regions. The results suggest as season-dependent over-estimation of ICESat sea ice thickness by the Envisat product, an over-estimation which is particularly pronounced during February/March and May/June. The observed differences between the products are discussed in the light of potential causes.

General Comments: Continuing to inter-compare the ESA SICCI-2 sea-ice thickness data sets with independent sea-ice thickness observations to better evaluate these products certainly is very useful, contributing to our understanding of the uncertainties and limitations of these. Since a more focus has been on inter-comparison studies for the CryoSat-2 part of these data sets, this study is particularly welcome because it focusses on the Envisat period. Sea-ice thickness observations from that period (before March 2011) are notoriously sparse. The authors solved this by using upward looking sonar draft observations and a sea-ice thickness data set based satellite-based laser altimetry - which appears close to the best one can do and nicely expands on existing work carried out in the framework of the ESA SICCI-2 project. The manuscript would benefit from a number of substantial amendments to figures and text, potentially making the results more credible. The most important points I laid out in my general comments GC2 and GC3 below, accompanied by a suite of specific comments detailing the potential measures that could lead to an improved credibility of the results and to a mitigation of their potential misinterpretation. It is recommended that all co-authors adequately contribute with their expert knowledge to the manuscript.

Printer-friendly version

GC1 (editoral): Please be consistent with usage of total freeboard and sea-ice freeboard. Try to be as clear as possible in the way you assign the respective wordings to the two different types of instruments used. 
GC2: The comparison with the ULS data requires more details and a slight change in the way the comparison is presented. Currently the message to be taken is based on very few data points with limited discussion of the reliability of such an intercomparison. This bears the risk to over-interprete the results which to my opinion should be avoided. Please see my specific comments.

GC3: The discussion of the results of the comparison between Envisat and ICESat SIT data sets (i.e. Section 4) would benefit from some more considerations and re-writing. This applies to the quality of the ICESat data considered as "the truth", to the way the statistical results are interpreted, and to some details in the description how the comparison was carried out. Please see the respective specific comments.

Specific Comments: Abstract: - I recommend to include number of the comparison results between the two altimeter sea-ice thickness data sets and the ULS draft data set, i.e. something like the mean difference and its standard deviation. It would also be helpful to learn from the abstract rightaway whether you transferred the ULS draft measurements into sea-ice thickness or whether you converted the satellite data into sea ice draft by simply saying whether you compared draft or sea-ice thickness. Why? Because any conversion (draft to thickness or thickness to draft) has inherent errors. I recommend to focus on the one parameter, e.g. the mean difference and its standard deviation, and report this for the entire Antarctic and all sub-regions. This gives a more complete picture of your intercomparison than the bits and pieces of smallest deviations, largest correlations, largest deviations, etc. you do provide currently.

Lines 63-64: - "Hendricks et al 2010" $\rightarrow$ I guess there is more recent literature in peer-reviewed journals targeting this issue and even using for improvement of current retrievals? Please check. - "Ku-Band radar altimeters" $\rightarrow$ Is this a special feature of Ku-Band or perhaps generally valid for radar altimeters? Please change accordingly if needed.

Lines 65-67: - There is more recent literature about this issue - perhaps Kern and

Printer-friendly version

Discussion paper
Interactive

comment 
Ozsoy-Cicek 2016: Satellite Remote Sensing of Snow Depth on Antarctic Sea Ice: An Inter-Comparison of Two Empirical Approaches; or Kern and Ozsoy 2019: An attempt to improve snow depth retrieval using satellite microwave radiometry for rough antarctic sea ice; or Price et al. 2019: Snow-driven uncertainty in CryoSat-2-derived Antarctic sea ice thickness - insights from McMurdo Sound - "must use" $\rightarrow>$ no, not necessarily. There are other alternatives to deal with snow, e.g. using snow accumulation from re-analyses.

Line 77: Please already here add one sentence about ICESat-2 which is in orbit since September 2018 and provides excellent freeboard measurements which are not discontinuous in time anymore.

Line 85: This approach was not suggested by Worby. It was suggested by Kern et al. 2016. This approach has been called 1-layer Worby et al approach in Kern et al. (2016) because the ratio between snow depth and sea-ice thickness used in Eq. 2 is based on the ASPeCt observations published by Worby et al. (2008); Please correct accordingly. Note that this approach has been further refined by Li et al. 2018 (see comment under "typos/editoral issues" Lines 50-52), who derived first guess values of snow depth and sea-ice thickness directly from ICESat data using empricial approaches instead of the climatological values used by Kern et al. (2016); that way the sea-ice thickness retrieval using this 1-layer method is certainly considerably more accurate than the one introduced for the first time in Kern et al. (2016) because it takes the actual situation into account better. Of course, it needs to be noted that the empirical approaches used by Li et al. (2018) were in turn developed from a (more or less comprehensive) suite of historic in-situ observations of freeboard, snow depth and sea-ice thickness which in a way have the character of a climatology as well.

Lines 98-107: - The last paragraph of this block about Envisat should come first and it should be expanded. Please provide a bit more information about the Envisat RA2 (time of operation, orbit details (observation hole at pole?), nominal resolution, altimeter type etc.). Then you could come up with something along the lines how sea-ice

Printer-friendly version

Discussion paper
Interactive comment 
freeboard is obtained and then how from the sea-ice freeboard sea-ice thickness is derived. I suggest to avoid this "is defined" in Line 99; I don't think there is a definition. What the RA2 measures is a runtime which is converted into a surface elevation which in turn is converted into a radar freeboard in case of sea ice. For this freeboard it is not precisely known whether this is representative of the location of the ice-snow interface. It is assumed that this is the case based on the experience from laboratory work (you cited it, Beaven et al.) and because of this snow-depth dependent radar signal delay is applied to convert the radar freeboard into what is assumed (not defined) the sea-ice freeboard. - Line 101: Kern et al. (2016) is certainly not the correct reference here. That paper is about ICESat and has nothing to do with radar altimetry. You hence need to find another paper. Since you have at least two radar altimeter experts, which were involved in the derivation of the Envisat data set you are using, among your co-authors it would be easy to find an appropriate reference. Just ask them! - Equation 1: Please explain in the text what the rhos are. - Lines 103/104: I am sure the information about how the sea-ice thickness is retrieved is given in the respective ATBD of the data set; again I recommend to ask the two people who were actively involved in the processing of the Envisat data set. You will need this information also for answering which sea-ice concentration product is included in the data set (Line 110) and for figuring out what the sea-ice concentration threshold is (anyways) which was used to derive freeboard and sea-ice thickness (Line 108).

Line 131: 60\%: Why do you use a different sea-ice concentration treshold for the ICESat data than you use for the Envisat data?

Lines 133-139: - I suggest to add a bit more information about i) the field of view of such a sensor; ii) the temporal resolution with which the data are provided and which you use for your comparison; iii) the accuracy. - I would not group ULS data into "in-situ" (Line 135) because also ULS technique is kind of remote sensing and not an in-situ measurement such as a drilling of snow stake measurement would be. - The sentence containing "the most suitable criterion to evaluate" should possibly be rewritten along

Printer-friendly version

Discussion paper
Interactive comment 
the lines that such data are particularly well suited to evaluate the temporal evolution of the sea-ice thickness at a fixed location. How well their accuracy is (suited) we don't know (yet). And such data are not overly well suited to evaluate the spatial distribution of the sea ice thickness. Also, when using ULS data sea-ice motion as to be taken into account. - What are the assumption behind the empirical equation 4 - or in other words: Where lie its limitations of applicability / inherent uncertainties?

Interactive comment

Lines 141-148: "humidity" The amount of humidity in the atmosphere is certainly a impacting the quality of the Envisat RA2 measurements but is to my knowledge taken into account by the on-board microwave radiometer being sensitive to the atmospheric water vapor load. While I would not exclude that the humidity of the atmosphere could also have an impact on snow wetness or the available of free (liquid) water within the snow I an inclined to say that its effect is much smaller than the direct influences of both the air temperature / surface temperature and the net surface radiation flux. These parameters directly determine snow melt - be at the surface or internally, i.e. within the snow. Above a surface or snow temperature of $-5 \mathrm{deg} C$ the liquid water fraction in the snow increases. I would therefore suggest to look for air or surface temperature rather than humidity and also look at the net surface radiation. The relative humidity is a derived, secondary quantity which is possibly also more prone to errors than the temperature itself.

Line 151-153: - "For each period ... If ICESat data has overlapping time ..." -> Perhaps better: "We use Envisat data of every month which overlaps for at least ten days with a particular ICESat measurement period. For some ICESat measurement period this requires to average over Envisat sea-ice thickness data of two months (see Table 1)." - I note that period ON2005 starts October 21 and hence has 11 days in October with data while Envisat data are only taken from November. Please fix.

Printer-friendly version

Figure 1: I suggest to replace "sea ice freeboard" with "freeboard" in the caption of this figure to underline that you are targeting to illustrate both, total freeboard and sea-ice freeboard. 
Figure 2: - Did you create this map on your own or is this taken from the publication / data website? If the latter you either need to get the permission or at least state that you modified if from ... - What are the numbers in the white boxes referring to? - Please provide a table with the respective operation times of the ULS which overlapped with your combined ICESat - Envisat sea-ice thickness data set. If I recall correctly not all of these were in operation all the time. - Please write what the background information is ... it is bathymetry.

Figure 3: - The lines denoting the borders between the different regions is hard to see and should be made thicker or plotted using a different color. - Specify what the white grid cells stand for.

Lines 159-166: - How were ULS data co-located with the satellite data products? Also, I note that Envisat is on EASE grid but ICESat is on polar-stereographic grid. Which one did you choose? - What do you mean by "absolute" in Line 159? - "in situ" -> ULS is not an in situ measurements. - What does "-6" "-5" appended behind the ULS station numbers mean? - I don't think "deformed ice" and "thin ice" are particularly well chosen because also thin ice can be deformed. It might be better to refer to old or perennial ice in case of the location close to the peninsula - albeit local polynyas might produce an applicable amount of seasonal ice as well - and predominantly seasonal ice in case of the Eastern Weddell Sea.

Lines 167-181 / Figure 4: - It appears to me that the number of ICESat values used in this comparison are so small that it is almost impossible to provide an adequate statistics. In order to make the comparison look at bit more reasonable I suggest to combine the two successive records for 229 and 231, i.e. plot time series for the combined data set 229-5 and 229-6 as well as 231-6 and 231-7. That way you would not be forced to draw conclusions from just two data pairs. In addition, it appears to me that you computed RMSD values based on this completely different statistics and I suggest that you add a comparison between ULS data and Envisat data at the same sampling in time provided by ICESat. In that case you would need to specify

Printer-friendly version

Discussion paper
Interactive comment 
whether you'd average over May and June in the Envisat product to obtain a value representing the ICESat May/June measurement periods. - Please adequately note in the text that you take uncertainty information from the data sets, i.e. that both data sets, the ICESat and the Envisat one provide uncertainty information based on the retrieval. In this context you need to take into account that the uncertainty of the Envisat is possibly reduced by a factor of $1 / \mathrm{sqr}(\mathrm{n})$ with $\mathrm{n}$ being the number of grid cells used to compute the $100 \mathrm{~km}$ mean gridded version of the Envisat sea-ice thickness. - I suggest to rewrite the full paragraph once you have this third set of more sparsely sampled Envisat data. Please when re-writing it provide systematically the information about the range of differences and the RMSD for all data sets, i.e. ICESat - ULS, Envisat - ULS, Envisat_sparse - ULS for 207, 229 5+6, 231 6+7 - ideally in a table. I would not over-interprete grid cells with "open water" in the satellite products because values might simply be missing due to other factors. In your discussion of these results you should remind the reader that ULS is a measurement device obtaining very local data of sea ice which passes / develops over it - in your case - over the course of a month. My question is: how accurately did you take the exact measurement period of ICESat into account here? Did you average over the, e.g. 35 days of a period or did you average over the calendar months? In addition: you compare one sea-ice thickness value from a $100 \mathrm{~km} \times 100 \mathrm{~km}$ large grid cell with a value representative of a footprint of several 10 meters (I assume). This has an influence on both the sea-ice thickness as well as your estimate of the open water fraction obtained to carry out the comparison using the grid-cell mean sea-ice thickness.

Figures 5 to 7: - Please consider extending the latitudinal range further to the north so that you do not truncate the ice-covered area. - Please consider to show all maps with the same size. Currently, the maps for autumn are largest while those for summer are smallest. Having three maps in a row (as in Figure 7) seems to be a viable solution; hence, if you transpose rows to columns and show the years in rows rather than columns you can realize my suggestion. - I note that you show the two sea-ice thickness data sets at their native grid resolution while the difference map (of course)

Printer-friendly version

Discussion paper
Interactive comment 
is in the grid resolution of the ICESat product. Please make a note in the caption of Figure 5 to clarify that this is done on purpose. - I assume that white denotes missing data? This is fine (as long as stated once in the caption) for the sea-ice thickness maps but interferes with grid cells having a valid sea-ice thickness value in both data sets but a difference close to zero; in the difference maps the color white is not used uniquely - which I suggest to change. - I note a vertical line of grid cells without valid values just west of the date line, i.e. $180^{\circ} \mathrm{E}$. Please consider removing this / filling it with valid difference values. - Cosmetics: The color bar would be sufficient with half its horizontal width. Am I correct in assuming that the quantity displayed is sea-ice thickness or sea-ice thickness difference? Consider you want to uses these figures in a presentation - it cannot go without the caption the way you solved it now. If you would state along with the color bar which physical quantity is shown the figures would be self-explainable. You could combine this information with the information about the product which currently is provided left of the maps.

Table 2: - Please consider adding the standard deviation of the mean for the columns denoted "ENV $(\mathrm{m})$ " and "ICE $(\mathrm{m})$ ". Ideally, you do this after the interpolation of the Envisat data to the ICESat resolution.

Lines 186-202: - Line 186: Delete "And" - Line 187: Who is "They"? - Line 189-191: I don't find the spring maps clear enough to make the statement given about the Ronne Ice Shelf polynya. The other issue, that Envisat kind of fails to see the young in in the southern Ross Sea is something which is noted in the above-mentioned (by you) ESA-SICCI-2 project PVIR report; you might want to cite this here. - Line 191: I don't think that this recirculation of thicker ice from the Amundsen Sea into the Ross Sea is an anonamous phenomenon. You might consider removing this statement. - Line 193/194: What are "posivite" or "negative deviation" between Envisat and ICESat SIT? Perhaps it would be better to speak about "Envisat SIT exceeds ICESat SIT" ... and "Envisat SIT is below ICESat SIT" ... or similar. - Line 194-195: Isn't this statement a bit hypothetic? In the Arctic, for example, maximum SIT values are observed in April

Printer-friendly version

Discussion paper
Interactive comment

\section{(n)}


or even May with little change between April and May. In addition, since you compare grid-cell mean SIT values you might have a substantial influence of a potentially lower sea-ice concentration. It would be interesting to look at the pure SIT values, wouldn't it? - Line 195/196: Where is this statement substantiated from?

Lines 203-213: - Line 203: I note that FM-periods are at the end of summer and mark the begin of the freezing season; in 2007 this period is even shifted by one month towards winter (March-April). It might therefore be better to refer to this period as autumn or summer-autumn transition and denote the May/June period as winter. Or simply change the beginning to "At the end of summer melt (FM), ..." - Lines 208/209: "may result": Don't you have sea-ice concentration data provided along with the Envisat SIT data? You can simply check this out and make a more clear statement here. - Lines 209/210: "Envisat also detects the Ross sea ice ..." -> I guess you wanted to refer to the Ross Ice shelf polynya and the sea-ice thickness? - Line 211: See my previous comment about this formulation. - "is bad" $\rightarrow$ please change to something without valueing, e.g. "We find larger RMSD values ... by $X Y$ and smaller correlation values ... by $X Y$ than for spring.

Lines 214-224: - Line 215: Which "errors" are you referring to here? I guess you still refer to the differences between Envisat and ICESat SIT, right? So there is no error involved. - Line 218: Which "deviations"? To which panel of Figure 7 are you referring? I only find differences. - Line 219: "The ice growth ..." $\rightarrow$ I suggest to delete this sentence as it is not relevant in this context. - Line 221: "from to thinner" $\rightarrow$ "from thicker to thinner" - Line 223: "... are high among all periods ..." $\rightarrow$ I assume you wanted to write "highest among"? - Line 224: I suggest to add 1-2 sentences pointing out that correlations are substantially better for 2004 and 2005 than 2006 while, at the same time, the coverage with valid Envisat SIT data (Fig. 7, top row) is considerably smaller than for ICESat (Fig. 7, middle row). This needs to be discussed: two months for 2004 and 2005, including May, only 1 month (June) for 2006; since MJ periods are located in the seasonal upswing of sea-ice coverage and thickness it can make a big 
difference to include or exclude one month of data. I also suggest to point out the interannual variability in data pairs with valid data for each season. There a considerable variations in the number of valid data pairs within one season - mostly because of data gaps in the ICESat SIT data set.

Figure 8: - I suggest to re-order the panels to [end-of-]summer (left), winter (middle) and spring (right) to have the correct time line of development. This would ease discussion about whether mean (and modal?) SIT increase from winter to spring - which is what should be the case. - I am wondering about the coarse bin-size of 0.5 meters; did you experiment with smaller bin-sizes of, e.g. $0.25 \mathrm{~m}$ or $0.2 \mathrm{~m}$. If not, what is your motivation to use such a large bin size. Could that be the accuracy? - I suggest to use blue and red also as font colors for the annotation within the panels, make the rectangles in a) simple lines, and add the modal value, ideally both as a (dashed) vertical line and as a numner. Note that the modal SIT value is a better measure for thermodynamic sea ice thickness growth than the mean SIT. - Are the normalized to have sum 1?

Lines 225-238: - Line 226: "... agreement ... turns bad ..." -> please re-phrase and omit "bad". - Line 231: "Both of the two data sets cannot detect the thin ice smaller than $0.5 \mathrm{~m} \mathrm{..."} \rightarrow$ Looking at Fig. $8 \mathrm{a}$ ) and b) reveals that there are very small but non-zero fractions of SIT values y $0.5 \mathrm{~m}$. I suggest to rewrite: "We find that thin ice with thickness values $<0.5 \mathrm{~m}$ is practically absent in both investigated products." In order to avoid the conclusion that ICESat or Envisat are not capable to resolve such thin ice you might want to add a statement into that direction. - Lines 232-235: This passage should be re-written. It is not clear what you are referring to here. Of course the mean (!) SIT is larger in summer than winter or spring because any young ice or thin ice is missing and the only grid cells left with sea-ice coverage contain - at that time of the year which is actually end-of-melt - thick ice; this is comparable to the situation in the Arctic during the sea ice minimum in September. One should not refer to "growth" or "grow" here. What is more interesting is that the mean SIT derived with ICESat increases from MJ to ON periods while the Envisat one does not. As commented in the context of Figure 
8 I also encourage to include modal SIT values into the discussion. I recommend to delete the "The rest can be ..." sentence. - Line 236: "weaker" -> "smaller"; in addition you mention warm and wet weather condition and flooding sometimes. Does this refer to summer only or to the entire year? You might want to specify the key issue for penetration depth here which is wet snow as a consequence of, for example, abovefreezing air temperatures - Line 237/238: "Since ICESat ..." $\rightarrow$ This sentence should be deleted because it is wrong. ICESat is much better suited to detect thin ice than Envisat thanks to its small footprint size.

Lines 239-251: - I suggest to delete the very first sentence. It is fine to simply also show results broken down by region as is commonly done in many publications about sea-ice concentation and thickness products. - I find this paragraph not particularly well structured. Perhaps you can solve this by avoiding to jump between the different statistical parameters but stick with one and describe it in a comparative manner across the regions / seasons - ideally highlighting the most interesting issues. Clarification what you mean by "deviations" would also help - see respective previous comments. - Lines 248-251: I suggest to delete these sentences as they appear to be too hypothetical. Also, you start the discussion in the next section anyways.

Figure 9: - I suggest to add the identity line in each scatterplot.

Table 3: - I suggest to re-order seasons such that one can clearly see the change in SIT from autumn (or winter as l'd call it) to spring. - Please add a unit for RMSD. I possible please provide standard deviations for the means of ENV and ICE (like I suggested already in the context of Table 2).

Lines 253-262: Title of this section: "deviation" -> l'd say "deviation" is used if one wants to describe how well a data set agrees to a known standard or calibration data set. This is not the case in your study. You inter-compare different data sets of which none is the standard. Therefore the term "difference" would match considerably better. - I suggest to delete the first sentence of this paragraph. You don't need this kind of

Printer-friendly version

Discussion paper 
introduction here. - Line 259/260: "respond differently to different surface roughness." $\rightarrow$ This is true ... sure ... but how about resolving leads / open water required for an adequate representation of the local sea-surface height required to derive the freeboard? Isn't this a much more important difference in the obervation capabilities of the two sensors? - Line 261: "... while ICESat uses ..." $\rightarrow$ This reads as if the ICESats sea-ice thickness values are not retrieved using the hydrostatic equilibrium assumption ... but for both sensors this assumption is applied; ONE of the differences is in fact the treatment of the snow depth - here you are correct.

Figure 10: I have the same comments as I voiced in the context of Figures 5 to 7 in terms of latitudinal extent, the vertical line of missing data just west of $180 \mathrm{E}$ and the fact that the Figure cannot be used on its own because the annotation of the color bar is not complete.

Figure 11: - The maps in this figure would need to be changed according to changes noted for Figure 10. - Relative humidity is typically given in percent, i.e. your scale would go from $70 \%$ to $100 \%$ and would be entitled "Relative humidity [percent]". - Do you think these values are credible? I can agree with the fringe of low values around the continent, indicating the impact of the catabatic air flow ... but why is the relative humidity higher over sea-ice covered areas than over open water areas? Isn't open water a much more efficient source of evaporation and hence input of water vapor into the lower troposphere? The only reason I could think of why the relative humidity is higher over sea ice than open water is because of the colder temperatures. I note that the difference between open water and sea ice is most pronounced for the cold May/June period compared to the milder October/November period. This is kind of in line with my argumentation. - It does make a difference whether one uses saturation with respect to a water or to an ice surface when computing the vapor pressure and hence the relative humidity. Which of these is used for the relative humidity you plot here?

Lines 264-278: - Line 268: "weaken" -> "decrease"; "into the snow-ice interface" -> C13

Printer-friendly version

Discussion paper
Interactive

comment 
"into the snow" - In the context of what is written here and in the paragraph Line $279++$, I recommend to read » Kwok and Kacimi, 2019: Three years of sea ice freeboard, snow depth, and ice thickness of the Weddell Sea from Operation Icebridge and CryoSat-2 and the paper by » Paul et al., 2018: Empirical parametrization of Envisat freeboard retrieval of Arctic and Antarctic sea ice based on CS-2: progress in the ESA CCI . These papers are good preparations for a thorough discussion of the issues touched upon. - Lines $271++$ : I don't want to rule out that the atmospheric humidity might have had an impact on Envisat RA2 measurements that differs from the impact on ICESat measurements. However, I would expect that this would involve the entire troposphere and not the near surface relative humidity. I am sceptical, however, whether the near surface humidity, which is second order parameter, is a good measure to judge whether a snow surface / cover is wet or dry. I would have thought that the air-temperature is the more direct driver. Please check.

Lines 279-284: - Line 280 "footprint is rather rough" $\rightarrow>$ I don't understand. How can a footprint be rough? The ice or snow surface can be rough. - That the detection of leads can affect the detection of thinner ice sounds trivial. If a sensor cannot resolve leads and/or resolve leads only indirectly and potentially also at the wrong position, then it is more than likely that thin ice thickness is not retrieved overly well. But what has this to do with "shorter ice"? What is this? What is "wider sea ice". I suggest to completely rewrite this paragraph after having studies the two paper I just recommended to read. Also, and this is what I cannot understand: You have two experts for radar altimetry based sea-ice thickness retrieval among your co-authors - so two people you could ask directly.

Lines 286-302: - I suggest to repeat one more time a) Envisat SIT is based on an AMSR-E snow-depth climatology and b) ICESat SIT does not use snow depth in the retrieval but uses climatological ratios between snow depth and sea-ice thickness in form of the factor $\mathrm{R}$ to compute the modified density. This is a lot of "meat" for discussion. $\rightarrow$ pitfalls of using a climatology instead of actual snow depth values (Bunzel and

Printer-friendly version

Discussion paper
Interactive comment

Discussion paper

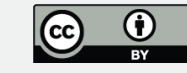


Notz, 2018, Retrievals of Arctic sea-ice volume and its trends significantly affected by interannual snow variability); $\rightarrow$ reduction of the relative uncertainties by using a climatology rather than actual values; $\rightarrow$ limitations of the R-factor usage in the ICESat SIT data set (also based on climatology, and in addition based on ASPeCt observations with their known difficulties); $\rightarrow$ discussion how the new approach by Li et al. (2018, mentioned above) would compare here; discussion of when which snow physical properties would cause which bias for which of the periods considered (i.e. winter compared to spring compared to summer). - Line 299/300: Kwok and Maksym (2014, see above) looked into this and figured out what typical snow depths appear to look like and Kern and Ozsoy-Cicek (2016, see above) figured out that indeed there is a substantial under-estimation. I recommend to refer to numbers from the respective publications to underline your assumptions and hypotheses. - Line 200/301: The sentence "Therefore, the sea ice ... negative." needs to be rewritten. - Line 302: "snow depth uncertainty over the deformed sea ice" $\rightarrow$ Please be more specific. You know which sign this uncertainty would have (a positive bias!); hence, cases with actually a lot of deformed ice would have too little snow retrieved while cases with just a bit deformation would have fairly good retrievals. But: The ENV data set uses a climatology. Therefore, the largest effect might not come from deformed / level ice's impact on the snow-depth retrieval but the largest effect might be due an actual snow depth which differs completely from that represented by the climatology - no matter whether the sea ice is particularly deformed or not.

Lines 318-325: This paragraph would read better in Section 4 I suggest.

Typos / editoral issues: Line 11: "global" -> "hemispheric" because you consider the Antarctic not the entire Earth.

Line 16: Suggest to add "these" between "with" and "field" to underline that the results of your comparison are only based on the ULS data.

Printer-friendly version

Line 42: "errors" -> l'd prefer to see "limitations" 
Line 43: "AEM data" $\rightarrow$ Please tell the reader which parameter is derived from the AEM data.

Line 44: "only exists in the Weddell Sea" -> this is not correct. Please change to: "has mostly been obtained in the Weddell Sea"

Line 45: - Why "time series"? These observations are not carried out at a specific point. - "freeboard observation" $\rightarrow$ Yes, but there is an additional snow radar which allows additional snow depth estimates. Also, you might want to include the information whether the freeboard measured is the total or the sea-ice freeboard.

Line 46: "limited to several trajectories in the Weddell Sea" $\rightarrow$ This is not entirely true since there are also trajectories in the Bellingshausen Sea; more importantly, however, is that substantial work has been done with these data, e.g. Kwok and Maksym, 2014: Snow depth of the Weddell and Bellingshausen sea ice covers from IceBridge surveys in 2010 and 2011: An examination; and Kwok and Kacimi, 2019: Three years of sea ice freeboard, snow depth, and ice thickness of the Weddell Sea from Operation Icebridge and CryoSat-2; this work the the importance of this data for our understanding of sea-ice thickness retrieval of Antarctic sea ice using altimeter data should not be undervalued.

Line 47: ULS data provide primary the sea-ice draft; sea-ice thickness is a derived variable. I suggest to change this to draft.

Line 48: "the basin ..." $\rightarrow>$ "a basin ..."

Line 50-52: - Bernstein et al. (2015) -> I suggest to add Li et al. 2018: Spatio-temporal variability of Antarctic sea-ice thickness and volume obtained from ICESat data using an innovative algorithm

Printer-friendly version

- I suggest to remove passive microwave and SAR here to remain concise and then stress that altimetry has proven to currently be the best source for Antarctic wide seaice thickness retrieval over the full thickness range. 
Line 55: "three"? -> I guess it is two decades because ERS1/2 is in progress but not yet ready to be released.

Line 58: "one of the latest ..." $\rightarrow$ Perhaps latest is not the predominant issue here but the fact that it is finally a combination of CryoSat-2 AND Envisat.

Line 71: "estimates ... depth)" $\rightarrow$ "allowed to estimate the total freeboard (sea ice freeboard + snow depth) via determination of the surface elevation"

Line 74: I suggest to write "compared" instead of "developed".

Line 76: "period" -> "periods"

Line 78-81 / 88 - Since you defined ULS already there is not need to again write it in full length. - The same applies to airborne electromagnetic EM sounding which should be "AEM sounding" in Lines 79. - I am sure the PVIR has an author team so you can refer to it as "author et al., 2018". - I am relatively sure that the evaluation this report refers to also included CryoSat-2 data. Please check and amend accordingly.

Line 96/97: The URL given only points to the Envisat sea-ice thickness data set; hence you need to remove the CryoSat-2 part of this sentence.

Line 97: "gridded" -> "grid"

Line 98: "consistent" -> why consistent? What do you mean by this?

Line 108: "girds" -> "grids"

Line 112: You have defined ICESat already, so no need to still use the full name.

Line 113: This is total freeboard, right? See GC1.

Line 116: "five main" -> "several"

Printer-friendly version

Line 119: "suggested by Worby" $\rightarrow$ see comment I had under "specific comments" already. 
Line 126: "ASPeCt observations" -> cite the respective Worby et al. (2008) paper here. Line 128: This is total freeboard, right? See GC1.

Interactive comment on The Cryosphere Discuss., https://doi.org/10.5194/tc-2020-48, 2020. 